\title{
Um Modelo de Predição para o Sucesso no Mercado Musical
}

\author{
Carlos V. S. Araujo \\ Universidade Federal do Amazonas \\ Instituto de Computação \\ Manaus, Amazonas 69080-900 \\ vicente@icomp.ufam.edu.br
}

\author{
Rafael Giusti \\ Universidade Federal do Amazonas \\ Instituto de Computação \\ Manaus, Amazonas 69080-900 \\ rgiusti@icomp.ufam.edu.br
}

\author{
Marco A. P. Cristo \\ Universidade Federal do Amazonas \\ Instituto de Computação \\ Manaus, Amazonas 69080-900 \\ marco.cristo@icomp.ufam.edu.br
}

\begin{abstract}
In this paper we present an ongoing research that aims to produce a model to predict success in the musical market. To reach this goal, it is necessary, initially, to identify influence factors in the market, which is the current focus of this research. In this branch, we identify that tweets have influence over the popularity of an album in Spotify. We also found out that Pop has more tendency to have the most popular song of a year, among other analyses. The next steps of this research are to list even more influence factors, generate the model using artificial neural networks and validate it with real-world cases.
\end{abstract}

\section{KEYWORDS}

Complex Network, Music Success, Artificial Neural Network

\section{INTRODUÇÃO}

A indústria do entretenimento (cinema, jogos, música e televisão) movimenta bilhões de dólares anualmente em todo o mundo. Segundo a Associação da Indústria de Gravação da América (Recording Industry Association of America - RIAA $)^{1}$, somente a indústria da música movimentou, em 2016, US\$ 7,7 bilhões nos Estados Unidos. Desse total, 75,5\% são referentes às músicas digitais (streaming ou download).

Uma forma de maximizar os ganhos de artistas, gravadoras e selos musicais seria utilizando um modelo de predição de sucesso de um produto no mercado musical (álbuns, singles ${ }^{2}$, DVDs etc.) Tal modelo poderia ser utilizado para se obter melhor resultado comercial, escolhendo a melhor época para seus lançamentos e uma melhor estratégia de marketing. A geração desse modelo e sua validação é o resultado final esperado ao fim dessa pesquisa em desenvolvimento. Para obtenção de tal resultado será necessário o uso de técnicas de mineração e fusão de dados, aplicadas a fatores de influência no mercado, tais como redes sociais, colaborações entre artistas e a popularidade do gênero musical.

As Redes Sociais Online (RSO), que vêm sendo adotadas por gravadoras para a divulgação de novos singles ou álbuns, são um desses fatores de impacto. Nessas RSO, os usuários têm a possibilidade de expor suas opiniões e percepções pessoais sobre os mais variados assuntos [16], sendo que estas podem vir a ser utilizadas como base

\footnotetext{
${ }^{1}$ www.riaa.com/

${ }^{2}$ Singles são músicas lançadas individualmente em que se espera obter um bom resultado comercial.

In: XVIII Workshop de Teses de Dissertações (WTD 2018), Salvador, Brasil. Anais do XXIV Simpósio Brasileiro de Sistemas Multimídia e Web: Workshops e Pôsteres. Porto Alegre: Sociedade Brasileira de Computação, 2018.

(c) 2018 SBC - Sociedade Brasileira de Computação.

ISBN 978-85-7669-435-9.
}

de influência para o comportamento de outros usuários [23], assim impactando na divulgação desses singles ou álbuns.

Trabalhos como os de Chen and Chellappa [5], Dewan and Ramaprasad [7] e Chen et al. [6] já mostram o impacto do conteúdo gerado por usuários em RSO no sucesso de um álbum musical.

Outro fator que vem sendo utilizado por artistas para obterem maiores vendas em suas músicas e álbuns são as colaborações, que ocorrem quando dois ou mais artistas diferentes participam de uma mesma música [3]. Trabalhos como os de Smith [22], Gleiser and Danon [12] e Uzzi [24] mapearam a colaboração de artistas em variados estilos musicais. Nesses trabalhos as colaborações foram mapeadas por meio de grafos, sendo os artistas representados como nós e suas colaborações por arestas.

A compreensão de como essas redes de relacionamentos são formadas e como evoluem ao longo do tempo possui elevado valor comercial. Esse conhecimento é fundamental para traçar eficazes estratégias de mercado para artistas consolidados e, principalmente, para novos artistas [3].

A popularidade do gênero musical no ano de lançamento de um álbum também é importante fator para sua popularidade. Mauch et al. [18] e Lopes [15] já estudaram como se dá o processo de popularização de um gênero, mostrando que revoluções são o fator principal para que um gênero passe a ter maior destaque. Mas, para que o interesse nele seja mantido é necessário que novos artistas de destaque nestes surjam a cada ano.

Utilizando esses fatores, além de outros ainda a serem estudados, é possível montar uma base de dados que pode ser usada para gerar um modelo de predição. Esse modelo se diferenciaria da literatura existente, pois em geral estes tratam apenas de fatores isolados, enquanto usaremos um englobamento desses elementos. Assim, buscando ser o mais próximo possível do valor real de sucesso alcançado pelo lançamento musical.

O restante deste trabalho está organizado da seguinte forma: na seção 2 são apresentados trabalhos relacionados ao desta pesquisa. Enquanto na seção 3 as contribuições esperadas a serem obtidas com esse estudo são mostradas. Na seção 4 os resultados obtidos e esperados são descritos. Por fim, na seção 5 está uma breve conclusão.

\section{TRABALHOS RELACIONADOS}

Os trabalhos de destaque nessa área de pesquisa voltaram-se a realizar predições utilizando-se de fatores isolados. Logo, nesta seção apresentamos alguns desses trabalhos e também aqueles que obtiveram fatores de influência como resultado, os quais podem vir a ser utilizados em nosso modelo.

$\mathrm{Na}$ área da análise de impacto de Redes Sociais Online no mercado musical, destacam-se os trabalhos de Dhar and Chang [9], 
Dewan and Ramaprasad [8] e Chen et al. [6] que serão explanados a seguir.

Dhar and Chang [9] examinaram a viabilidade de usar conteúdos gerados por usuários, em redes sociais e blogs, na predição de vendas na indústria musical. Para tal, utilizaram uma amostra de 108 álbuns e acompanharam as mudanças sobre o que estava sendo falado a respeito deles durante as quatro semanas que antecederam e sucederam os seus lançamentos. Como resultado, descobriram que as vendas estão correlacionadas com o volume de postagens em blogs sobre um álbum.

Dewan and Ramaprasad [8] traçaram um paralelo entre as novas mídias (downloads), mídias antigas (CD’s) e as vendas na indústria da música. Mais especificamente, estudaram a interação entre o que era comentando em blogs de distribuição gratuita de músicas, a quantidade de vezes que uma música era reproduzida na rádio e as vendas dessas músicas e de seus respectivos álbuns. Para análise, utilizaram a metodologia de autoregressão de vetores de painel (PVAR). Como resultados, demonstraram que o número de vezes que a música é tocada na rádio está diretamente relacionado a vendas futuras, tanto da música quanto do seu álbum.

No trabalho de Chen et al. [6], os autores empregaram o mesmo modelo utilizado por Dewan and Ramaprasad [8], a autoregressão de vetores de painel, com o intuito de investigar a relação entre divulgações em redes sociais e as vendas de música. Para isso, avaliaram os conteúdos automáticos e pessoais gerados na rede social MySpace $^{3}$ e descobriram que a transmissão em mídias sociais tem um efeito significativamente positivo nas vendas. Mais especificamente, mostraram que esse efeito era ainda maior quando eram excluídas da análise as mensagens automatizadas.

Em relação à análise do processo de colaboração entre artistas na área musical, os trabalhos de Grandadam [13], Giaquinto et al. [11] e Budner and Grahl [4] se destacam.

Budner and Grahl [4] propuseram uma rede englobando os vários profissionais da indústria musical. Para montá-las utilizaram-se da lista "500 maiores álbuns de todos os tempos" da revista Rolling Stones ${ }^{4}$ e o livro "1001 álbuns que você deve ouvir antes de morrer"[10]. Nessa rede, cada profissional representava um nó e estes eram conectados se tivessem trabalhado em um mesmo disco. Ao analisar a rede gerada, os autores identificaram que as profissões mais importantes da indústria musical, em termos de conectividade, são os artistas principais e engenheiros de som.

Já Grandadam [13] e Giaquinto et al. [11] montaram redes para o gênero Jazz. O primeiro analisou o selo musical Blue Note, conectando na rede os artistas que participavam de uma mesma sessão de gravação. Então, utilizando-se da distância geodésica e do coeficiente de clustering, identificou que os artistas mais famosos colaboravam com novos artistas para que o status do selo se mantivesse. Enquanto o outro trabalho, de cunho mais generalista, apontou que para um artista obter sucesso nesse gênero é mais importante que este saiba tocar múltiplos instrumentos do que ter um valor de centralidade alto em sua rede de colaboração.

$\mathrm{Na}$ área de análise de popularidade de gêneros musicais, Mauch et al. [18] procuram identificar quais os principais fatores que implicam na popularização desses gêneros. Para isso, coletaram $86 \%$

\footnotetext{
${ }^{3}$ https://myspace.com/

${ }^{4} \mathrm{http} / / /$ www.rollingstone.com/music/lists/500-greatest-albums-of-all-time- 20120531.
}

das músicas que apareceram no ranking "Billboard Hot 100"5 entre 1960 e 2010, onde as distribuíram em 13 diferentes grupos. Para tal, utilizaram as frequências de seus elementos, tanto instrumentais, quanto vocais. Por fim, os autores afirmam que a popularidade dos gêneros musicais é determinada por revoluções. Citam três destas como as de maior relevância: a invasão britânica de 1964, o movimento New Wave de 1983 e a ascensão do Rap em 1991, sendo essa última a de maior importância por ter mudado a estrutura musical americana.

Enquanto, Lopes [15] estuda a quantidade de novos e antigos músicos na lista de 100 singles mais populares entre os anos de 1969 a 1990. Apresentam que por ano ao menos $29,5 \%$ dos singles no Top 100 anual são de novatos, valor que chega até a 45\%. Enquanto, aqueles com mais de oito anos de carreira representam no máximo $15,9 \%$. Esses dados mostram que os novos artistas são fundamentais para a popularidade dos gêneros musicais.

Um outro aspecto a se considerar é o impacto das letras para o sucesso de uma música. Destaca-se aqui o trabalho de Marzagão [17], que analisa as letras de músicas brasileiras de oito diferentes gêneros musicais. Nesse trabalho, cada artista estudado recebe um número referente ao seu vocabulário, dado por uma razão entre a quantidade de palavras únicas utilizadas em suas composições e o valor total de músicas desse artista. O autor mostra que os artistas de Rap são os que tem maiores vocabulários em suas músicas, já os de Sertanejo tem o menor. Também chegou a duas conclusões: quanto menor o vocabulário do artista, maior tende a ser o seu sucesso; e, excluindo o Rap, quanto mais antigo é o artista, maior é o seu vocabulário.

Como foi apresentado, há uma gama de trabalhos que tratam de fatores de influência no mercado musical. Muitos desses trabalhos se atêm somente a mostrar que há esse impacto, não tentando prever qual seria o sucesso futuro de um álbum ou música a ser lançada. Já aqueles que realizam alguma forma de predição, utilizam somente fatores isolados. O diferencial da nossa proposta em relação aos trabalhos apresentados é o de englobar os vários fatores de influência, com o intuito de gerar um modelo de predição que seja o mais próximo possível do valor real de sucesso alcançado pelo produto musical.

\section{CONTRIBUIÇÕES ESPERADAS}

A grande contribuição esperada é voltada ao mercado musical, que utilizando-se do modelo poderá obter melhores rendimentos comerciais.

Entretanto, as contribuições acadêmicas serão advindas do processo requerido para a obtenção de tal resultado. Visto que serão necessárias técnicas de fusão e mineração de dados, computação social, aprendizagem de máquina e redes complexas.

Nesse quesito será primordial determinar a influência de parâmetros de influência no mercado musical que ainda não foram devidamente estudados, agregando conteúdo a literatura atual.

Por fim, o processo tomado para projetar o algoritmo que combine os resultados analíticos para determinação do modelo a ser proposto é significativo para a área de ciência da computação, mais

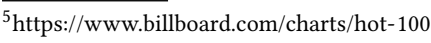


precisamente a de ciência dos dados. Visto que será necessário aplicarmos redes neurais artificiais e encontrarmos aquela que venha a obter o melhor aprendizado dado o conjunto de fatores estudados.

\section{RESULTADOS}

Nessa seção apresentamos os resultados já obtidos e o que esperamos alcançar no decorrer desta pesquisa de mestrado, estando contidas nas subseções os resultados 4.1 e 4.2, respectivamente.

\subsection{Resultados Obtidos}

Na primeira etapa da pesquisa buscamos identificar fatores de influência no mercado musical. Essa etapa gerou três artigos publicados que serão explanados nesta seção.

Em Araujo et al. [2] identificamos uma correlação entre mensagens no Twitter ${ }^{6}$ (também conhecidas como tweets) escritas uma semana antes e após o lançamento de um álbum e seu respectivo valor em popularidade no Spotify ${ }^{7}$. Esse valor é um número entre 0 e 100 gerado pela própria plataforma com base no número de streams das músicas desse álbum.

Utilizamos a seguinte metodologia: inicialmente coletamos as mensagens utilizando a API da própria plataforma. Após, realizamos um pré-processamento, onde deixamos somente as mensagens únicas. Então, realizamos a detecção da polaridade dessas mensagens. Finalmente, analisamos se havia correlação entre os dados coletados e geramos dois modelos de predição. Tal processo está ilustrado na Figura 1.

Para mostrar que havia correlação entre as mensagens e o valor em popularidade no Spotify dos álbuns, aplicamos os coeficientes de correlação de Pearson, Spearman e de distância. Observamos que os dados apresentavam correlação linear positiva quando considerávamos o total de tweets ou somente aqueles com polaridade positiva. Valores ratificados com o teste-t que apresentou confiança acima de $95 \%$ nos resultados obtidos.

Utilizando tais dados, geramos dois modelos de predição: um utilizando a quantidade total de tweets e outros somente aqueles com polaridade positiva. Esses modelos foram avaliados considerando o coeficiente de determinação, a variância residual e o erro quadrático médio. Ao analisar esses três fatores, identificamos que o modelo das mensagens positivas apresentou melhor resultado. Ele é dado por:

$$
P_{p o s}(p)=56,700+\left(6,389 \times 10^{-4}\right) p,
$$

onde $P_{\text {pos }}(\cdot)$ é a previsão da popularidade no Spotify, considerando a quantidade $p$ de tweets positivos.

Em Araujo and Nakamura [1] utilizando os rankings "Year-End Hot 100 Songs" ${ }^{8}$ da Billboard entre os anos de 1969 a 2017 buscamos identificar fatores de influência sobre a popularidade dos gêneros musicais. Essas listas apresentam as 100 músicas mais famosas de cada ano nos Estados Unidos.

Realizada a coleta das músicas das listas, foi necessário identificar o gênero de cada uma delas. Para isso geramos um algoritmo que

\footnotetext{
${ }^{6}$ https://twitter.com/

${ }^{7}$ https://www.spotify.com/

${ }^{8}$ https://www.billboard.com/charts/year-end
}

realiza consultas à Wikipedia ${ }^{9}$ extraindo essa informação. Ao montar representações contendo o número de músicas de cada gênero por ano, observamos os períodos nos quais havia uma repentina mudança nesses valores. Ao identificá-los, analisando informações sobre o mundo da música na época, conseguíamos extrair o motivo de tal acontecimento.

Assim, identificamos que o principal fator de influência sobre a popularidade de um gênero musical é o surgimento de um cantor, banda ou um movimento de destaque. Entretanto, em caso de músicos faz-se necessário que outros artistas também consigam obter notoriedade em anos próximos, fazendo com que o gênero consiga se manter em destaque. Caso isso não ocorra, possivelmente haverá um aumento repentino de popularidade, mas que não consegue se manter, dificilmente alcançando mais que cinco anos de notabilidade.

Também observamos que a migração de artistas para outros gêneros e situações trágicas, como assassinatos, também são fatores, mas em menor grau.

O gênero Pop apresentou importante particularidade nessa pesquisa, pois a música mais popular de um ano tende a ser desse gênero. Esse fato foi observado mesmo em períodos onde o Pop não apresenta destaque na quantidade total de músicas.

Enquanto em Araújo et al. [3] buscamos entender como se dá o processo de surgimento de comunidades dentro de um gênero específico, nesse caso o Rap. Para isso, delimitamos a pesquisa a um escopo específico dentro desse cenário, que são as colaborações entre artistas nos álbuns do DJ Khaled, famoso artista americano conhecido por ter sempre vários músicos colaborando em suas músicas (uma delas conta com 12 colaboradores).

Montamos nossa base de dados a partir de uma coleta feita utilizando a API do serviço de streaming Napster ${ }^{10}$. Feita essa coleta, identificamos os músicos dessa base e montamos representações onde cada um deles era um nó, que eram ligados se haviam participado de uma mesma música. Os pesos dessas arestas foram dados pela quantidade de músicas em que apareciam conjuntamente. Montadas as redes era possível, então, analisá-las. Essa metodologia está apresentada na Figura 2.

Nesse trabalho, montamos nove redes da seguinte forma: a primeira tinha as colaborações do primeiro disco do Khaled, a segunda as referentes ao primeiro e segundo discos e assim sucessivamente. Logo, a nona rede, ilustrada na Figura 3, englobava todas as colaborações do artista no período contemplado na nossa base. Utilizamos a medida de betweenness centrality para identificarmos as comunidades destas, assim extraindo informações de relevância.

Somente foi possível dar significado às comunidades identificadas a partir da rede com as colaborações dos seis primeiros álbuns (essa rede conta com 109 nós e 313 arestas). Ao final, seis comunidades representativas foram identificadas, sendo uma delas a dos novos artistas (em cinza na Figura 3), o que significa que, no caso estudado, eles apresentam maior tendência a colaborar entre si do que com artistas de renome.

Em suma, os principais fatores que identificamos e que estão presentes em nossas publicações são:

\footnotetext{
${ }^{9}$ https://en.wikipedia.org/

${ }^{10}$ https://us.napster.com/
} 


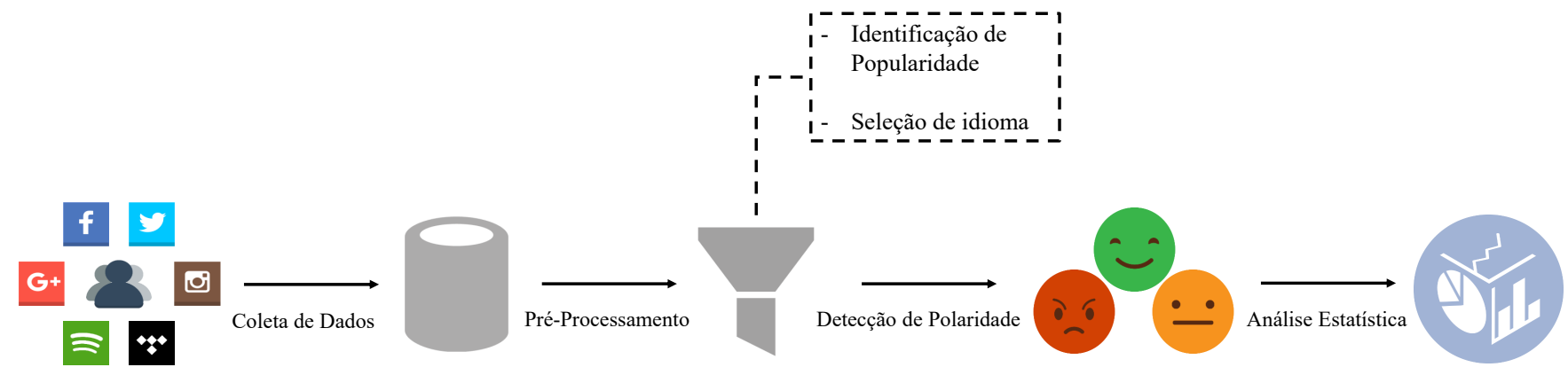

Figura 1: Metodologia utilizada em Araujo et al. [2].

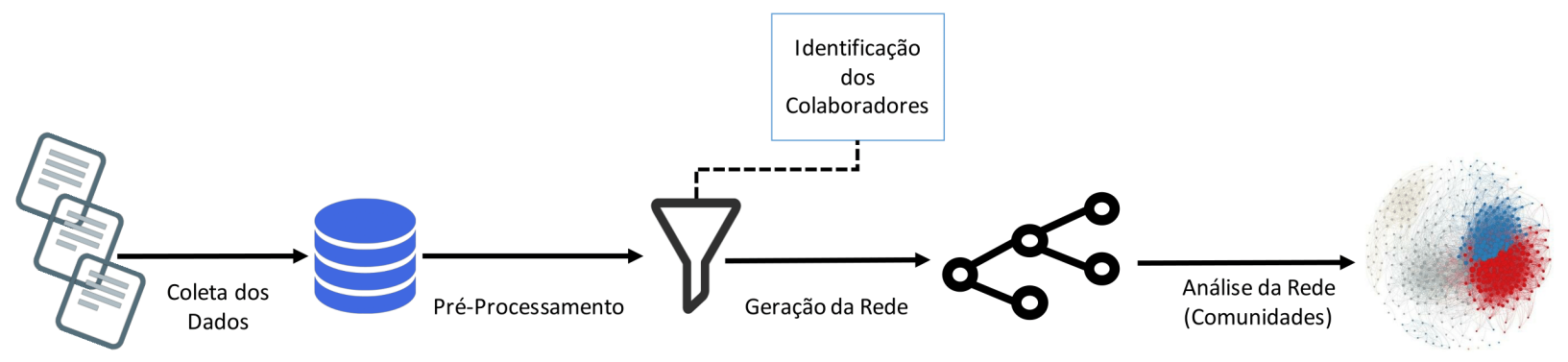

Figura 2: Metodologia utilizada em Araújo et al. [3].

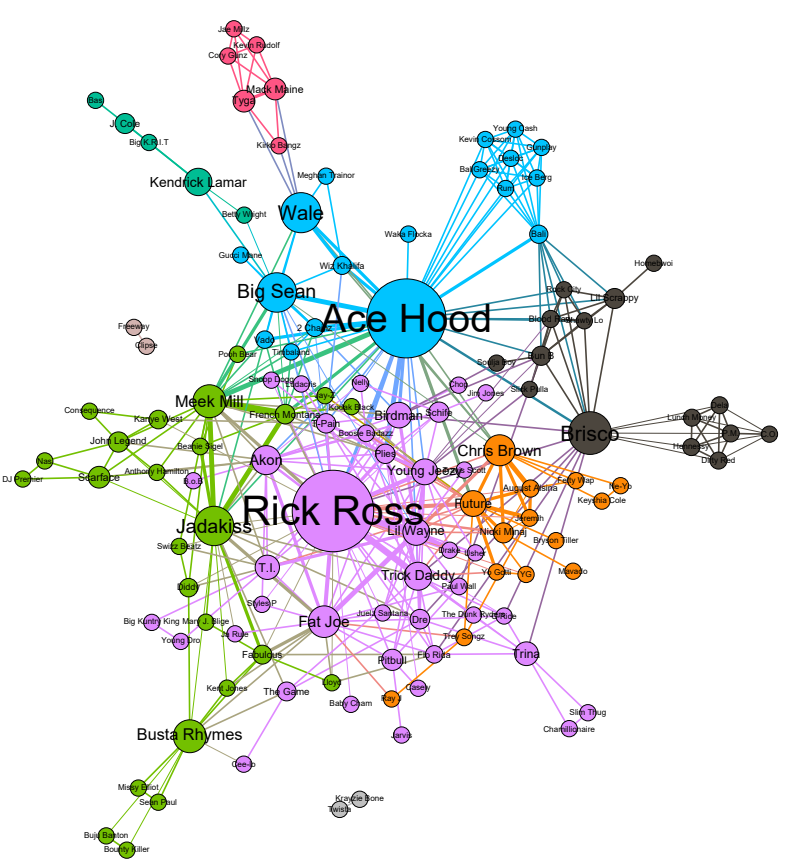

Figura 3: Rede contendo as colaborações entre músicos nos nove primeiros discos do DJ Khaled, extraída de Araújo et al. [3].
(1) Mensagens com polaridade positiva no Twitter tem impacto sob a popularidade de um álbum no Spotify;

(2) O surgimento de um ato musical ou um movimento de destaque é o principal fator de influência sob a popularidade de um gênero musical;

(3) Um artista ou banda podem alavancar sozinhos a popularidade de um gênero por um período de até cinco anos;

(4) O Pop é o gênero que apresenta maior tendência a ter a música mais popular de um ano;

(5) No Rap, novos artistas não apresentam tendência a colaborarem com artistas consagrados.

\subsection{Resultados Esperados}

Apesar de já termos obtidos alguns fatores em nossa pesquisa, consideramos que eles ainda não são suficientes para gerarmos um modelo com boa taxa de acerto, pois alguns dos parâmetros estudados tratam apenas de gêneros específicos. Para contornar esse problema devemos buscar generalizar todos os resultados obtidos, mostrando sua validade ou não em um âmbito mais geral.

Além disso, outros fatores ainda necessitam serem estudados. Para que seja possível encontrar entre uma gama de subconjuntos destes, aquele que apresente melhor resultado para utilizarmos em nosso modelo.

Assim, já está planejado um estudo voltado à verbosidade de artistas e sua relação direta com a popularidade destes. Apesar de Marzagão [17] já ter apresentado que quanto maior o vocabulário, menor o sucesso, esse resultado foi apresentado somente para as músicas brasileiras. Além disso, o parâmetro de sucesso difere daquele que planejamos utilizar. Nesse trabalho a colocação nas listas 
do site Vagalume ${ }^{11}$ foi usada como indicador, enquanto nós planejamos utilizar o Spotify, que é uma plataforma com abrangência mundial.

Logo, buscaremos uma relação entre o número de palavras distintas utilizadas por um artista em um novo lançamento e o valor em popularidade deste alcançado no Spotify. Podemos gerar um modelo de predição utilizando tal relação como parâmetro único e os analisando estaticamente de forma similar ao que foi feito em Araujo et al. [2].

Também percebemos que álbuns lançados em 2018 por alguns artistas de renome, tais como Beyoncé, Kanye West, Kid Cudi, The Chainsmokers, dentre outros, possuem menos de 10 músicas. Esse fato pode vir a indicar uma nova tendência no mercado musical, buscando adaptar-se às mudanças na forma do consumo de música. Esse fator ainda carece de estudo, onde iremos observar se há relação entre a quantidade de músicas em um álbum e a sua respectiva popularidade. Uma possível correlação entre a duração em minutos e a popularidade também poderia ser obtida como resultado desse estudo.

Com base nesses fatores de influência já obtidos em nossas pesquisas e de estudos publicados por outros pesquisadores, bem como outros que podem ainda vir a ser levantados em nossos estudos, produziremos um modelo de predição, de acordo com a metodologia ilustrada na Figura 4.

O modelo de predição será uma rede neural artificial [25]. O processo de treinamento de uma rede neural artificial é um processo de aprendizado automático no qual obtemos um modelo capaz de representar um conhecimento. Em nosso caso, o conhecimento desejado é a relação entre os fatores previamente estudados e o sucesso ou fracasso de um álbum. Redes neurais artificiais têm sido utilizadas com sucesso para a resolução de diversos problemas que envolvem predição. Por exemplo, para reconhecer atividade humanas [26], realizar diagnósticos clínicos [14] e predizer falência de empresas [19].

Um importante aspecto do emprego de estratégias de aprendizado de máquina para tarefas de predição é a definição de um conjunto adequado de atributos de entrada. Assim, iremos estudar a utilidade dos diferentes subconjuntos de fatores (atributos) na geração de modelos preditivos. Consideramos também a possibilidade de empregar modelos de aprendizado profundo [21]. Tais modelos são redes neurais artificiais recorrentes e se destacam por sua capacidade de automaticamente aprender uma representação dos dados originais, que frequentemente produz resultados superiores ao que seriam obtidos pela seleção manual de conjuntos de atributos para representação dos dados. Avaliaremos diversos modelos de predição, os quais serão comparados através de técnicas de validação cruzada [20].

O método a ser proposto fará sua predição para o valor em popularidade no Spotify uma semana após o lançamento do álbum ou single.

Em resumo, os próximos passos dessa pesquisa são:

(1) Generalizar os fatores de influência obtidos que são específicos de um gênero, mostrando sua validade ou não para os outros;

\footnotetext{
${ }^{11}$ https://www.vagalume.com.br/
}

(2) Identificar outros fatores ao analisar a verbosidade, número de faixas, duração, dentre outros elementos de um álbum;

(3) Utilizando redes neurais artificiais gerar um modelo que utilize de tais fatores para realizar a predição;

(4) Validar tal modelo com casos do mundo real.

\section{CONCLUSÃO}

Apresentamos aqui um projeto de mestrado em andamento que tem como objetivo propôr um algoritmo de predição da popularidade de um álbum no mercado musical. Mais precisamente o valor que esse álbum alcançará em popularidade na plataforma de streaming Spotify uma semana após o seu lançamento.

No atual estado de nossa pesquisa estamos analisando possíveis fatores de influência no mercado, visto que esses fatores serão o cerne do modelo que iremos propor.

Em nossos estudos já identificamos que mensagens com polaridade positiva no Twitter influenciam no mercado musical. E, também, que o surgimento de uma banda ou artista de sucesso pode alavancar sozinha a popularidade um gênero por um período de até cinco anos. Mostramos que revoluções musicais são o principal fator de influência sob a popularidade de um gênero. Apresentamos que o gênero Pop possui maior tendência a ter a música mais popular de um ano e que artistas novos não tendem a colaborar com os já consagrados no Rap[1] [2] [3].

Os próximos passos são elencar ainda mais fatores e utilizando-se de redes neurais artificiais, buscar um subconjunto de fatores e pesos que entregue o melhor modelo de predição. Esse modelo gerado será testado e validado com casos do mundo real, onde buscaremos atingir a maior taxa de acerto possível. Assim, estabelecendo um valor base para estudos futuros nessa área.

\section{AGRADECIMENTOS}

Os autores agradecem ao CNPq pelo financiamento dessa pesquisa.

\section{REFERÊNCIAS}

[1] Carlos V.S. Araujo and Eduardo F. Nakamura. 2018. Identification of Most Popular Musical Genres and their Influence Factors. In Proceedings of the 24nd Brazillian Symposium on Multimedia and the Web (WebMedia '18). ACM, New York, NY, USA, 4. https://doi.org/10.1145/3243082.3264665

[2] Carlos V.S. Araujo, Rayol M. Neto, Fabiola G. Nakamura, and Eduardo F. Nakamura. 2017. Predicting Music Success Based on Users' Comments on Online Social Networks. In Proceedings of the 23rd Brazillian Symposium on Multimedia and the Web (WebMedia '17). ACM, New York, NY, USA, 149-156. https://doi.org/10.1145/3126858.3126885

[3] Carlos V.S. Araújo, Rayol M. Neto, Fabiola G. Nakamura, and Eduardo F. Nakamura. 2017. Using Complex Networks to Assess Collaboration in Rap Music: A Study Case of DJ Khaled. In Proceedings of the 23rd Brazillian Symposium on Multimedia and the Web (WebMedia '17). ACM, New York, NY, USA, 425-428. https://doi.org/10.1145/3126858.3131605

[4] Pascal Budner and Joern Grahl. 2016. Collaboration Networks in the Music Industry. arXiv preprint arXiv:1611.00377 (2016)

[5] Conny Chen and Ramnath Chellappa. 2009. Music sales and online user activity at social networking sites. In fifth symposium of the Heinz College's iLab Center for Digital Media Research, Carnegie Mellon University on Statistical Challenges in Electronic Commerce Research, Pittsburgh. 30-31.

[6] Hailiang Chen, Prabuddha De, and Yu Jeffrey Hu. 2015. IT-enabled broadcasting in social media: An empirical study of artists' activities and music sales. Information Systems Research 26, 3 (2015), 513-531.

[7] Sanjeev Dewan and Jui Ramaprasad. 2012. Research Note-Music Blogging, Online Sampling, and the Long Tail. Information Systems Research 23, 3-part-2 (2012), 1056-1067. https://doi.org/10.1287/isre.1110.0405

[8] Sanjeev Dewan and Jui Ramaprasad. 2014. Social media, traditional media, and music sales. Mis Quarterly 38, 1 (2014), 101-121. 

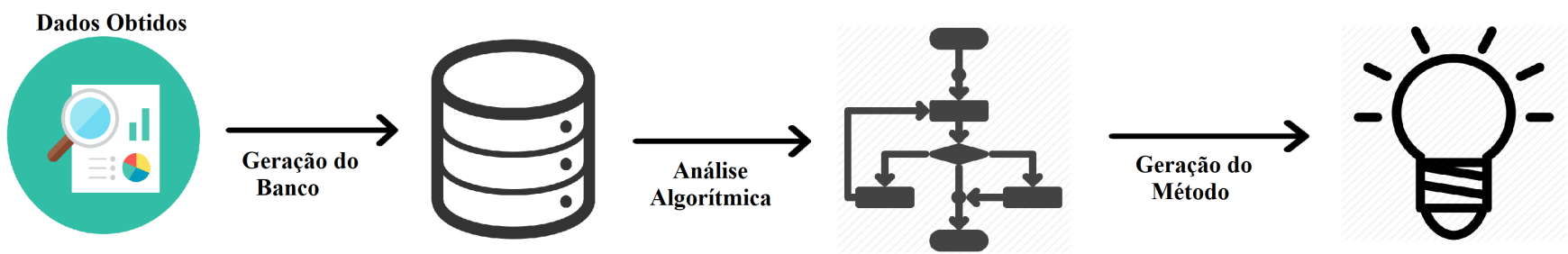

Figura 4: Metodologia proposta para a geração do método de predição.

[9] Vasant Dhar and Elaine A. Chang. 2009. Does Chatter Matter? The Impact of User-Generated Content on Music Sales. Fournal of Interactive Marketing 23, 4 (2009), 300 - 307. https://doi.org/10.1016/j.intmar.2009.07.004

[10] Robert Dimery. 2007. 1001 discos para ouvir antes de morrer. Tradução Carlos Irineu da Costa e Eliane Azevedo. Rio de faneiro: Sextante (2007).

[11] Gabriele Giaquinto, Cora Bledsoe, and Brian McGuirk. 2007. Influence and similarity between contemporary jazz artists, plus six degrees of kind of blue. Ph. D. Dissertation, Citeseer (2007).

[12] Pablo M Gleiser and Leon Danon. 2003. Community structure in jazz. Advances in complex systems 6, 04 (2003), 565-573.

[13] David Grandadam. 2008. Evolving networks and the finest in jazz. In Creative Industries and Intellectual Property Conference, Vol. 22

[14] Zachary Chase Lipton, David C. Kale, Charles Elkan, and Randall C. Wetzel. 2015. Learning to Diagnose with LSTM Recurrent Neural Networks. CoRR abs/1511.03677 (2015). arXiv:1511.03677 http://arxiv.org/abs/1511.03677

[15] Paul D. Lopes. 1992. Innovation and Diversity in the Popular Music Industry, 1969 to 1990. American Sociological Review 57, 1 (1992), 56-71. http://www.jstor. org/stable/2096144

[16] Rayol M Neto, Bruno Ábia, Thais Almeida, Fabíola Guerra Nakamura, and Eduardo Nakamura. 2017. Uma Abordagem para Identificação de Entidades Influentes em Eventos Comentados nas Redes Sociais Online. In Proceedings of the 14th Simpósio Brasileiro de Sistemas Colaborativos.

[17] Thiago Marzagão. 2015. text-analyzing Brazilian music. (2015). http:// thiagomarzagao.com/2015/12/24/text-analyzing-brazilian-music/

[18] Matthias Mauch, Robert M. MacCallum, Mark Levy, and Armand M. Leroi. 2015. The evolution of popular music: USA 1960-2010. Roya Society Open Science 2, 5 (2015). https://doi.org/10.1098/rsos.150081 arXiv:http://rsos.royalsocietypublishing.org/content/2/5/150081.full.pdf

[19] M. D. Odom and R. Sharda. 1990. A neural network model for bankruptcy prediction. In 1990 IfCNN International foint Conference on Neural Networks. 163-168 vol.2. https://doi.org/10.1109/IJCNN.1990.137710

[20] Stuart J Russell and Peter Norvig. 2010. Artificial Intelligence: a Modern Approach Pearson Education Inc.

[21] Jürgen Schmidhuber. 2015. Deep learning in neural networks: An overview. Neural Networks 61 (2015), 85 - 117. https://doi.org/10.1016/j.neunet.2014.09.003

[22] Reginald D Smith. 2006. The network of collaboration among rappers and its community structure. Fournal of Statistical Mechanics: Theory and Experiment 2006, 02 (2006), P02006.

[23] Bruno A Souza, Thais G Almeida, Alice AF Menezes, Fabíola G Nakamura, Carlos Figueiredo, and Eduardo F Nakamura. 2016. For or against?: Polarity analysis in tweets about impeachment process of brazil president. In Proceedings of the 22nd Brazilian Symposium on Multimedia and the Web. ACM, 335-338.

[24] Brian Uzzi. 2008. Collaboration Networks and Innovation: The Problem of Clustering. (2008).

[25] Marcel van Gerven and Sander Bohte. 2017. Editorial: Artificial Neural Networks as Models of Neural Information Processing. Frontiers in Computational Neuroscience 11 (2017), 114. https://doi.org/10.3389/fncom.2017.00114

[26] Jianbo Yang, Minh Nhut Nguyen, Phyo Phyo San, Xiaoli Li, and Shonali Krishnaswamy. 2015. Deep Convolutional Neural Networks on Multichannel Time Series for Human Activity Recognition.. In Ijcai, Vol. 15. 3995-4001. 